\title{
The Liverpool-Edinburgh high proper motion catalogue ${ }^{\star}$
}

\author{
R. S. Pokorny ${ }^{1}$, H. R. A. Jones ${ }^{1}$, N. C. Hambly ${ }^{2}$, and D. J. Pinfield ${ }^{1}$ \\ 1 Astrophysics Research Institute, Liverpool John Moores University, Twelve Quays House, Egerton Wharf, \\ Birkenhead CH41 1LD, UK \\ 2 Wide Field Astronomy Unit, Institute for Astronomy, School of Physics, University of Edinburgh, Blackford Hill, Edinburgh, \\ EH9 3HJ, UK
}

Received 5 January 2004 / Accepted 31 March 2004

\begin{abstract}
We present a machine selected catalogue of 11289 objects with proper motions exceeding $0.18^{\prime \prime} \mathrm{yr}^{-1}$ and an $R$-band faint magnitude limit of 19.5 mag. The catalogue was produced using SuperCOSMOS digitized $R$-Band ESO and UK Schmidt Plates in 287 Schmidt fields covering almost 7000 square degrees ( 17\% of the whole sky) at the South Galactic Cap. The catalogue includes UK Schmidt $B_{J}$ and $I$ magnitudes for all of the stars as well as 2MASS magnitudes for 10447 of the catalogue stars. We also show that the NLTT is $\sim 95 \%$ complete for Dec $>-32.5^{\circ}$.
\end{abstract}

Key words. stars: kinematics - stars: statistics - stars: subdwarfs - Galaxy: solar neighbourhood - stars: late-type stars: low-mass, brown dwarfs

\section{Introduction}

A number of recent studies have shown the power of proper motion surveys, particularly when combined with infra-red photometry, to identify various disk and halo populations, accurately characterize the nearby stellar census, identify nearby brown dwarfs, find common proper motion binaries and pick out members of kinematic moving groups. We briefly summarise below some recent studies in these areas.

Reid \& Cruz (2002), Reid et al. (2002), Cruz \& Reid (2002) and Reid et al. (2003) used NLTT data cross-referenced with the 2MASS Point Source Catalogue to search for nearby stars, both for follow up observation of specific stellar types, and for determination of the luminosity function, the mass-luminosity relationship, the stellar contribution to the local mass density, the velocity distribution and the stellar multiplicity statistics.

Rojo \& Ruiz (2003) used 112 stars taken from three areas of the Calan-ESO survey (Ruiz et al. 2001), identified with medium resolution spectroscopy, determined their Galactic velocities and constructed luminosity functions for the disk and halo populations using the $1 / V_{\max }$ method.

Reyle et al. (2002) took the sample of 693 stars from Scholz et al. (2000) and identified 301 of these stars in the DENIS catalogue. They used the $I-J$ colour to estimate the distances of these stars and found that at least 107 of them were within

Send offprint requests to: R. S. Pokorny,

e-mail: rsp@astro.livjm.ac.uk

* The full Table 1 is only available in electronic form at the CDS via anonymous ftp to cdsarc.u-strasbg.fr $(130.79 .128 .5)$ or via http://cdsweb.u-strasbg.fr/cgi-bin/qcat?J/A+A/421/763
$50 \mathrm{pc}$, including 15 within $25 \mathrm{pc}$ and one star, LHS124, which could be at $7.2 \mathrm{pc}$. Lepine et al. (2002) report the discovery of an M 8.5 star with proper motion $\mu=2.38^{\prime \prime} \mathrm{yr}^{-1}$ at a distance of $13.9 \pm 3.5 \mathrm{pc}$. Lepine et al. (2003) also reports the discovery of over 30 more stars within 25 pc. These studies enhance the local census of stars and enable more accurate determinations of the luminosity functions of both the disk and halo populations.

Salim \& Gould (2002, 2003; and Gould \& Salim 2003) have obtained improved astrometry and photometry for stars in the NLTT with Dec $>-17^{\circ}$ by matching that catalogue with the Hipparcos, Tycho-2 \& Starnet catalogues for bright stars, and the USNO-A and 2MASS catalogues for fainter stars. This revision to the NLTT has allowed them to improve the characterization and statistics for the NLTT.

Silvestri et al. (2002) took a sample of 13 white dwarf $\mathrm{M}$ dwarf high proper motion binaries with what they describe as "halo-like" velocities. 12 of these $13 \mathrm{M}$ dwarfs have nearsolar metallicity, from which they conclude that these systems are part of the kinematic tail of the thick disk population. They further state that this shows that the white dwarf components are not, therefore, part of the Galactic dark matter halo.

Ribas (2003) has suggested that the high proper motion field brown dwarf LP 944-20 is probably a member of the Castor moving group. Assuming this to be the case allows an age and a metallicity to be applied, which means that the properties of LP 944-20 may be compared to models.

An object which is present in our catalogue is a third component to the M dwarf white dwarf binary LHS4039/LHS4040 
which Scholz et al. (2004) recently identified as an active M 8.5 dwarf.

In this paper we expand on the previously published first release of the Liverpool-Edinburgh High Proper Motion Catalogue (Pokorny et al. 2003, hereafter PJH03). The aim of the project was to produce an internally homogeneous large area survey with well defined completeness limits. We chose to use only the two $R$-band datasets in order to prevent losing faint objects with extreme colours which would not show up in a search using the $B_{J}$ and $I$ band plates. PJH03 discussed the importance of proper motion surveys and described the survey data, search technique and the statistical tests conducted on the survey. We now present the fully completed version of the Liverpool-Edinburgh High Proper Motion Catalogue, update our analysis of the completeness levels for the survey and present 2MASS photometry for the catalogue sources.

Section 2 summarises the proper motion measurements and the scanned photographic plate magnitudes. Section 3 details how we cross matched our proper motion sources with the 2MASS database. Section 4 discusses ongoing and planned future work with the catalogue.

\section{The catalogue}

\subsection{Proper motion data}

We have now completed the survey and catalogue, using a further $156 R$-band Schmidt plates digitised by the SuperCOSMOS machine at the Wide Field Astronomy Unit of the Royal Observatory Edinburgh (Hambly et al. 1998, 2001a,b,c). The complete catalogue contains 11899 objects (approximately twice that of the first catalogue release) with proper motions exceeding $0.18^{\prime \prime} \mathrm{yr}^{-1}$ and $R$-band magnitude $\leq$ 19.5 mag in both plates, of which 610 are secondary or subsequent detections giving 11289 separate objects. Multiple detections are left out of all but one of the plots in this paper. The full survey used 287 Schmidt fields with plate centres at and below $-20^{\circ}$ declination covering almost 7000 square degrees ( $\sim 17 \%$ of the whole sky). This comprises all of the available sky out of the Galactic plane south of $-20^{\circ}$. Figure 1 shows the distribution of the fields used for the survey. Figure 2 shows the distribution of epoch differences for the $R$-band plates that were used for the survey, whilst Fig. 3 shows the distribution of high proper motion objects on an equal area plot of the sky. No fields with epoch differences of less than 3 years were used. The mean epoch difference is $\sim 8.5$. Almost all of the stars have $B_{J}$ and $I$ magnitudes, and 2MASS $J, H$ and $K_{\mathrm{S}}$ magnitudes (see Sect. 3). In the magnitude columns in Table 1 a value of -1.00 indicates that no plate was scanned in for that area and a value of 0.00 indicates that no object match was found in that passband.

In addition, the second release has updated $\sigma_{\mu}$ values, where we note that in the previous release we used an incorrect formula that generally overestimated these values. It should also be noted that the $R_{\mathrm{ESO}}, R_{\mathrm{UK}}$ and $I$ band magnitudes will differ slightly from those given by the SuperCOSMOS Sky Survey (SSS) (http://www-wfau. roe.ac.uk/sss/index.html). This is because the SSS magnitudes are updated as improved calibrations become available.

\subsection{Completeness}

As discussed in PJH03 a plot of $\log (\mathrm{Nc})$ against $\log (\mu)$ should have a slope of -3 for a complete sample of a population with constant space density. Figure 4 is such a plot for all nonmultiple detections in the catalogue. The least squares fit for this data has a slope of -3.057 . This gives us confidence that our search procedures, as described in $\mathrm{PJH} 03$, are robust.

Figure 5 is a histogram of the fractional proper motion errors $\left(\sigma_{\mu} / \mu\right)$ for all objects in the catalogue. It is truncated at 0.33 because we removed all objects with fractional errors greater than this value in order to minimize any false detections, although the binning means that it appears to truncate at 0.34 . We also visually inspected all objects with proper motions $\mu \geq 1.0^{\prime \prime} \mathrm{yr}^{-1}$. The simulated star completeness test detailed in PJH03 applies generally to the Schmidt plate data and the search process used for the whole of the survey. The results of that test are therefore applicable to the results for this second section of the survey. A version of the completeness test was conducted on a sample strip of fields running across the Galactic plane, to assess the minimum Galactic latitude it would be feasible to conduct the survey down to. The results indicate that although the completeness started to drop at $|b| \sim 35^{\circ}$, the search process would still run with reasonable efficiency to $|b| \sim 20^{\circ}$. The survey was therefore conducted on all available fields down to this limit. Table 2 shows how the relative completeness changes with low Galactic latitude, assuming that the number of proper motion stars is invariant with Galactic latitude, and the completeness for $|b|>$ $35^{\circ}$ is $95 \%$. As discussed in PJH03 the NLTT (Luyten 1979b) and LHS (Luyten 1979a) catalogues can be split into two areas; the northern area comprising all fields with Dec $\geq-30^{\circ}$ which are covered by the Palomar Observatory Sky Survey (POSS, Cabanela et al. 2003), and the southern area which comprises all fields with Dec $\leq-35^{\circ}$ which are covered by the Bruce Proper Motion Survey (BPM, Williams 2000). The drop in completeness shown in Table 2 can be seen in the plot comparing the number of stars in our catalogue to the number of stars in the NLTT for the northern POSS area of the NLTT (Fig. 6). The section of our catalogue which corresponds to the BPM survey area is still significantly more complete than the BPM (Fig. 7). For comparison Fig. 8 shows the number of stars in this catalogue and the NLTT for $-32.5^{\circ} \leq$ Dec $\leq$ $-17.5^{\circ}$ and $|b| \geq 40^{\circ}$. This shows that for $R \gtrsim 13$ this catalogue (for $|b| \geq 40^{\circ}$ ) and the NLTT (northern POSS section) contain almost exactly the same numbers of stars, and can therefore be assumed to have the same completeness in these areas. For this catalogue the completeness has been shown to be $~ 95 \%$ for $|b| \geq 35^{\circ}$, which suggests that the northern section of the NLTT (Dec $\geq-32.5^{\circ}$ ) is also $\sim 95 \%$ complete. Gould \& Salim (2003) use comparison with the Hipparcos and Tycho-2 catalogues to estimate the completenes of the NLTT as a function of Galactic latitude and find that it is $\geq 95 \%$ complete for $|b| \geq 20^{\circ}$ 


\section{Declination}

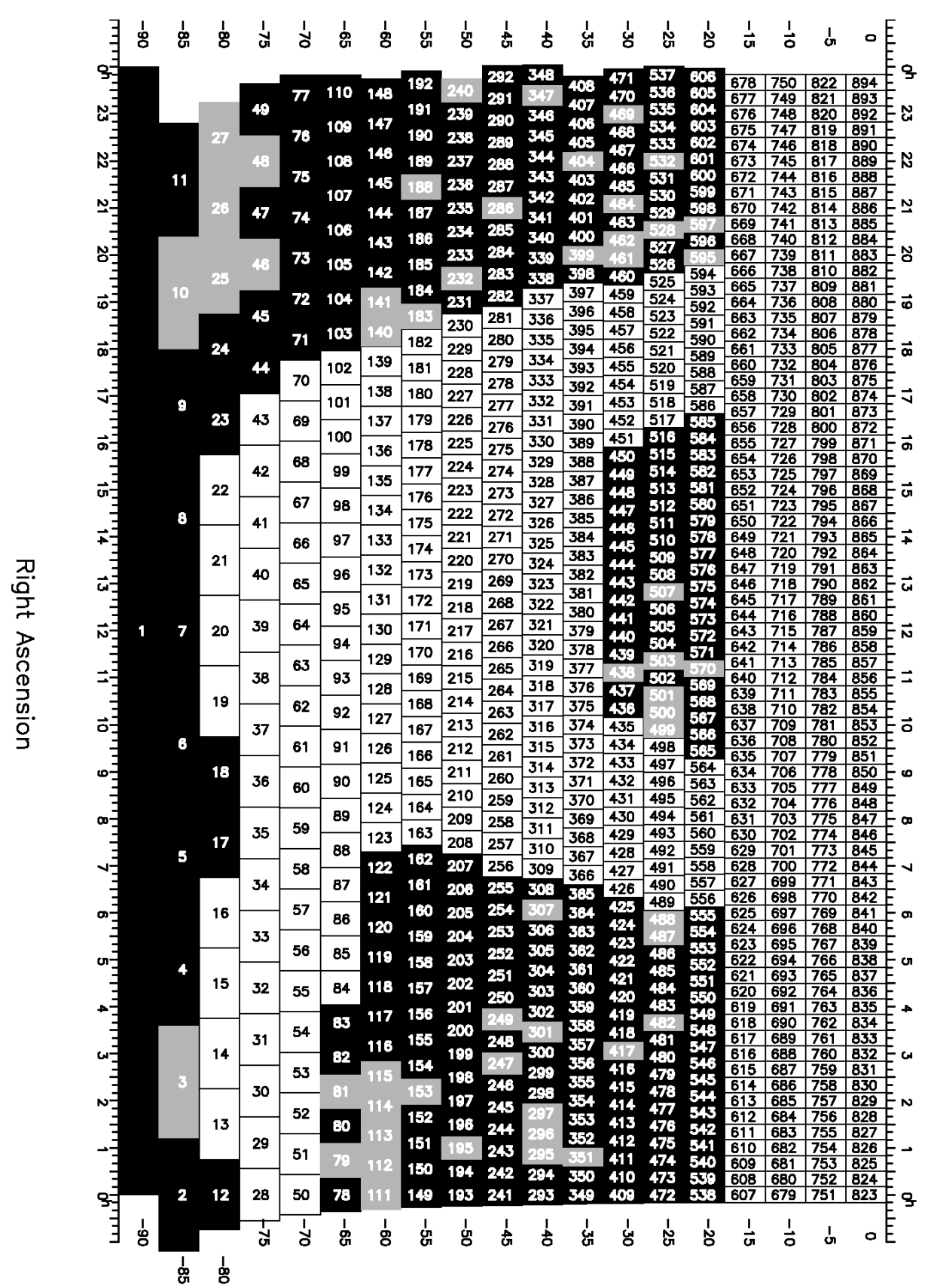

Fig. 1. Plot of the fields used in this survey. Fields shaded in dark were used, fields shaded in light were available, but could not be used either because of a short epoch difference between the ESO and UK plates or because they contained too few bright stars to calculate the full relative astrometry. Unshaded fields below Dec $=-20^{\circ}$ could not be used because they are too close to the Galactic plane or cover the Magellanic clouds.

and $V \lesssim 11.5$, which is in good agreement with our findings for fainter NLTT stars at mid and high Galactic latitudes.

\section{Cross matching with 2MASS}

We have used the All-sky-data-release of the Two Micron All Sky Survey (2MASS) Point Source Catalogue (Cutri et al. 2003), to provide near infrared photometry for our catalogue sources. 2MASS provides broadband $J, H$, and $K_{\mathrm{S}}$ photometry for point-like and extended sources over the full celestial sphere. The primary distinction between 2MASS and many other photometric systems is that the $K \mathrm{~s}$-filter transmission truncates at long wavelengths to avoid terrestrial $\mathrm{H}_{2} \mathrm{O}$ absorption (see Persson et al. 1998). The effective wavelength of the $K_{\mathrm{S}}$ filter is 2.15 microns (cf. 2.19 microns for standard $K$ filters). A secondary difference is in the $J$-band filter. The short and long wavelength cutoffs of this filter extend into the atmospheric $\mathrm{H}_{2} \mathrm{O}$ absorption features at 1.1 and 1.4 microns, 


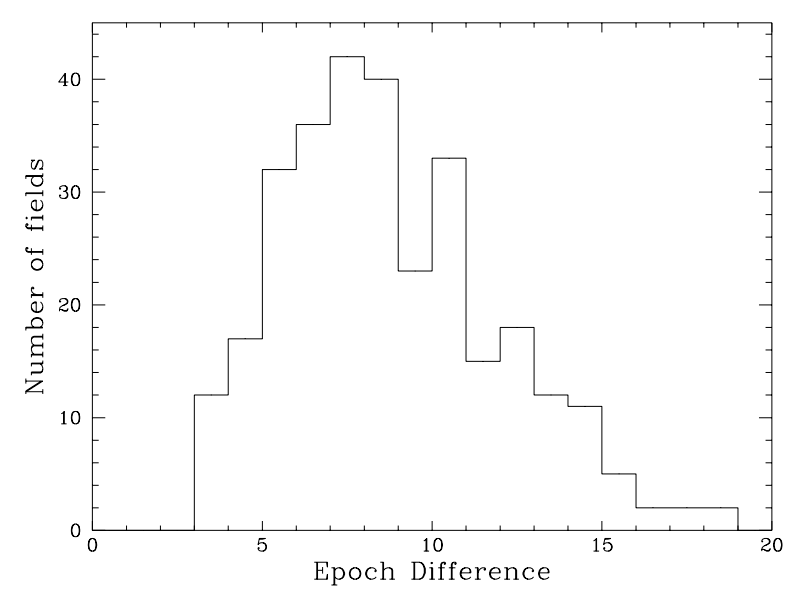

Fig. 2. Histogram of the epoch differences between the ESO and UK $R$-band plates for all of the fields used in the survey.

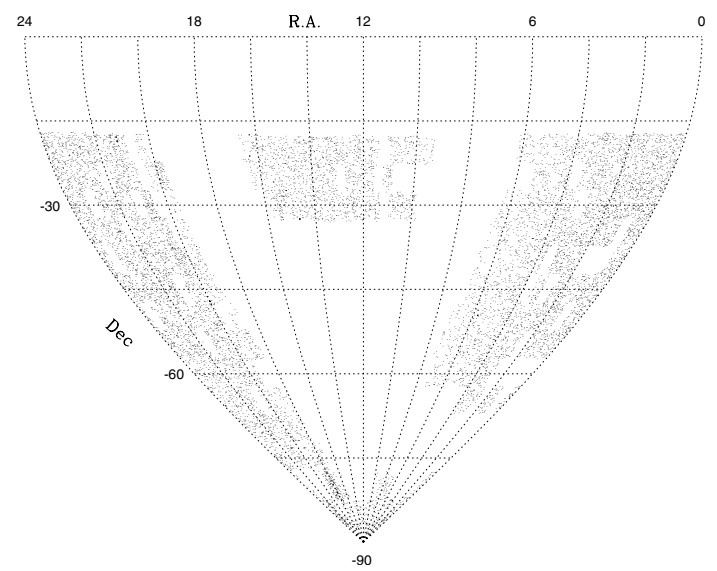

Fig. 3. Equal area plot of all objects in the catalogue.

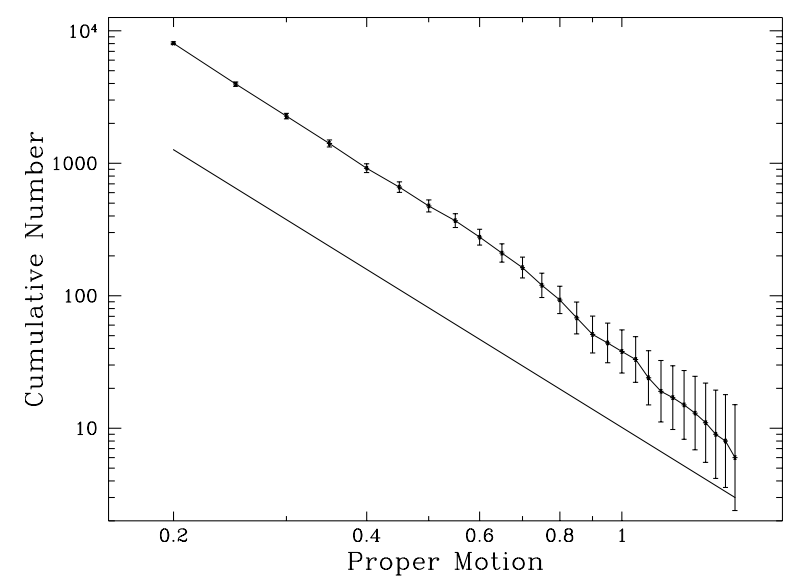

Fig. 4. Cumulative number of stars in this survey as a function of their proper motions. The offset straight line has a slope of -3 . Error bars are $\sqrt{ } \mathrm{N}_{\mathrm{C}}$.

which means that 2MASS $J$ magnitudes can be affected by atmospheric water vapour content (by up to $\sim 0.1 \mathrm{mag}$ ). Carpenter (2001) derives transforms between 2MASS magnitudes and those in other photometric systems, and finds only small differences between 2MASS $K_{\mathrm{S}}$ magnitudes and standard $K$ magnitudes. For a source to appear in the 2MASS catalogue, it must either have a signal-to-noise ratio $(S N R)>5\left(\sigma_{m}<0.22\right)$ in

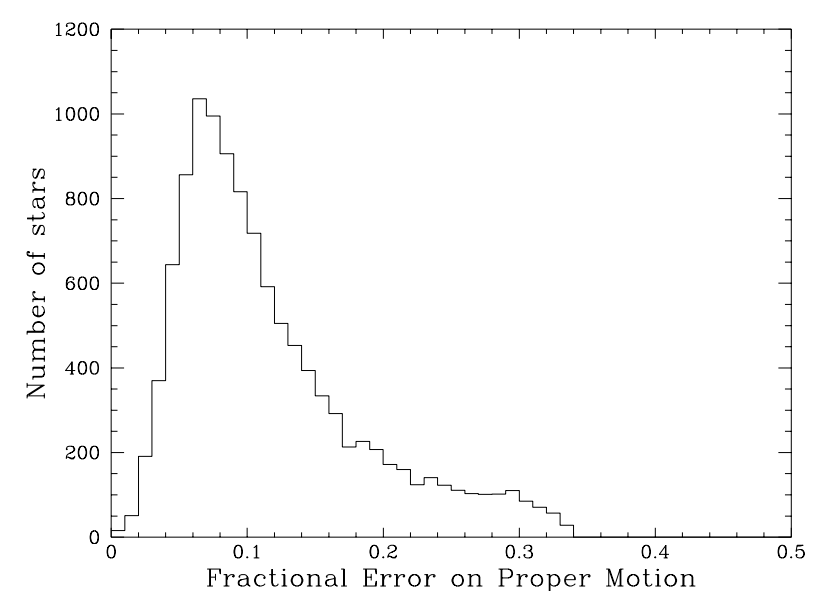

Fig. 5. Histogram of the fractional error on proper motion $\left(\sigma_{\mu} / \mu\right)$ for all entries in the catalogue.

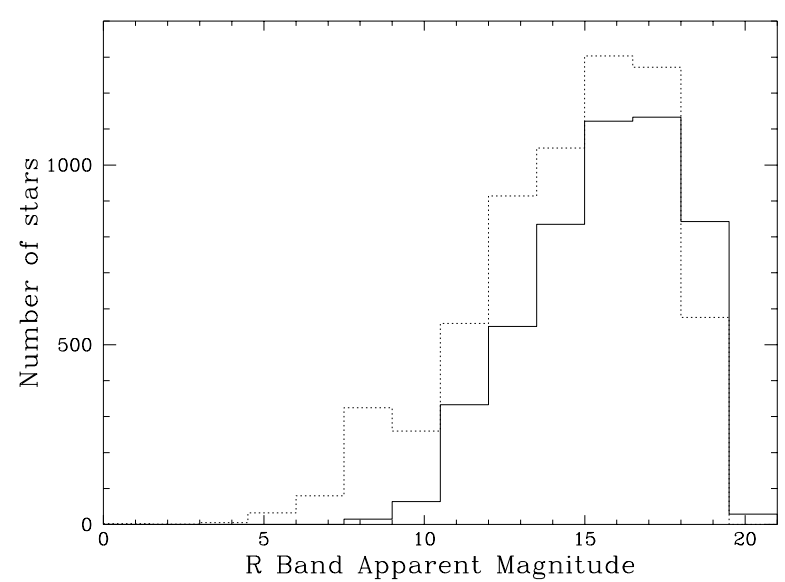

Fig. 6. Number of high proper motion stars per 1.5 magnitudes in $R$ for the Northern POSS areas of the NLTT: Solid line - this catalogue, dashed line - NLTT.

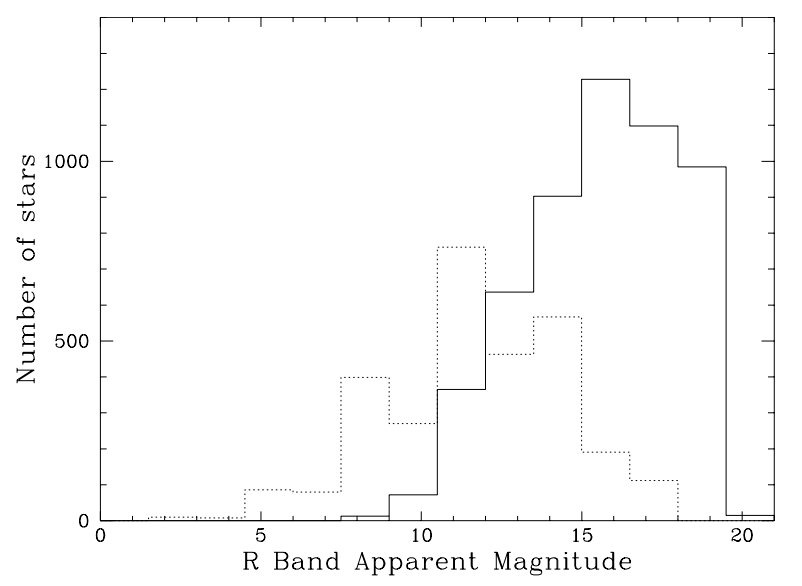

Fig. 7. Number of high proper motion stars per 1.5 magnitudes in $R$ for the Southern BPM areas of the NLTT: Solid line - this catalogue, dashed line - NLTT.

each band, or a $S N R>7\left(\sigma_{m}<0.16\right)$ in at least one band with fainter detections in the other(s). The 2MASS survey completeness limits are $J=15.8, H=15.1$ and $K_{\mathrm{S}}=14.3$. The $S N R=5$ limits are $J=17.2, H=16.2$ and $K_{\mathrm{S}}=15.8$. And the 
Table 1. Sample table from the full online catalogue, columns are; catalogue ID, RA, Dec (J2000), multiple detection flag, ESO/SRC field number, ESO $R$-band plate epoch, Epoch difference of observations, total $\mu$ (arcsec per year), error on $\mu$ (arcsec per year), position angle, magnitudes $-B_{J}, R_{\mathrm{ESO}}, R_{\mathrm{UK}}, I, J, H, K_{\mathrm{S}}$. For clarity four columns have been omitted, these are the components of the proper motion in RA and Dec and their errors. In the magnitude columns -1.00 indicates that no plate was scanned in for that area and 0.00 indicates that no match was found in that passband.

\begin{tabular}{|c|c|c|c|c|c|c|c|c|c|c|c|c|c|c|c|c|c|c|c|c|}
\hline$\overline{N_{\mathrm{C}}}$ & \multicolumn{3}{|c|}{$\overline{\mathrm{RA}}$} & \multicolumn{3}{|c|}{$\overline{\text { Dec }}$} & $\overline{F_{M}}$ & FFld & Epoch & Diff & $\bar{\mu}$ & $\overline{\sigma \sigma \mu}$ & $\overline{\mathrm{PA}}$ & $\overline{\overline{B_{J}}}$ & $R_{\mathrm{ESO}}$ & $\bar{c} R_{\mathrm{UK}}$ & $\bar{I}$ & $\overline{J J}$ & $\overline{\bar{H}}$ & $\overline{\bar{K}}$ \\
\hline $1-4081$ & 21 & 21 & 56.804 & -71 & 40 & 21.44 & 0 & 75 & 1984.650 & 6.15 & 0.35 & 0.04 & 131.44 & 16.24 & 13.99 & 14.11 & 12.39 & 11.24 & 10.65 & 10.42 \\
\hline $1-4082$ & 21 & 22 & 1.192 & -71 & 40 & 32.63 & 0 & 75 & 1984.650 & 6.15 & 0.35 & 0.04 & 130.05 & 16.16 & 13.94 & 14.10 & 12.24 & 11.24 & 10.65 & 10.38 \\
\hline $2-4478$ & 21 & 22 & 2.064 & -43 & 31 & 3.11 & 0 & 287 & 1989.740 & 3.88 & 0.19 & 0.04 & 226.97 & 20.21 & 18.13 & 18.11 & 17.14 & 16.41 & 15.58 & 15.72 \\
\hline $2-204$ & 21 & 22 & 2.514 & -22 & 59 & 32.08 & 0 & 530 & 1989.760 & 4.90 & 0.52 & 0.02 & 153.66 & 17.57 & 15.51 & 15.53 & 14.61 & 13.56 & 13.08 & 12.85 \\
\hline $1-4083$ & 21 & 22 & 13.769 & -69 & 32 & 8.61 & 0 & 75 & 1984.650 & 6.15 & 0.24 & 0.02 & 178.36 & 20.03 & 17.85 & 17.83 & 16.79 & 15.67 & 15.21 & 15.05 \\
\hline $2-4638$ & 21 & 22 & 14.160 & -43 & 59 & 11.59 & 0 & 287 & 1989.740 & 3.88 & 0.19 & 0.04 & 221.78 & 15.81 & 13.62 & 13.65 & 12.13 & 11.31 & 10.73 & 10.52 \\
\hline $2-699$ & 21 & 22 & 14.527 & -27 & 2 & 13.68 & 0 & 530 & 1989.760 & 4.90 & 0.35 & 0.05 & 177.75 & 13.40 & 12.31 & 12.35 & 11.74 & 11.38 & 10.95 & 10.84 \\
\hline $2-1383$ & 21 & 22 & 16.964 & -43 & 14 & 2.43 & 0 & 287 & 1989.740 & 3.88 & 0.28 & 0.05 & 173.05 & 14.26 & 11.70 & 12.06 & 10.01 & 9.13 & 8.53 & 8.21 \\
\hline $2-3291$ & 21 & 22 & 21.738 & -19 & 29 & 38.88 & 0 & 599 & 1987.490 & 4.26 & 0.21 & 0.05 & 204.95 & 15.68 & 13.86 & 14.01 & 13.11 & 12.77 & 12.20 & 12.09 \\
\hline $1-4084$ & 21 & 22 & 24.218 & -47 & 12 & 8.36 & 0 & 236 & 1985.766 & 6.88 & 0.39 & 0.06 & 165.01 & 16.38 & 14.07 & 14.30 & 12.03 & 11.09 & 10.53 & 10.26 \\
\hline $2-614$ & 21 & 22 & 24.247 & -47 & 12 & 9.60 & 1 & 287 & 1989.740 & 3.88 & 0.36 & 0.05 & 160.30 & 16.39 & 14.22 & 14.44 & 12.40 & 11.09 & 10.53 & 10.26 \\
\hline $2-5657$ & 21 & 22 & 30.959 & -33 & 27 & 45.66 & 0 & 402 & 1984.650 & 10.09 & 0.18 & 0.01 & 154.56 & 20.68 & 18.33 & 18.64 & 15.98 & 14.30 & 13.77 & 13.50 \\
\hline $1-4085$ & 21 & 22 & 40.096 & -71 & 22 & 48.29 & 0 & 74 & 1985.411 & 8.28 & 0.18 & 0.03 & 156.29 & 14.02 & 11.79 & 11.97 & 10.39 & 9.43 & 8.74 & 8.53 \\
\hline $2-456$ & 21 & 22 & 49.556 & -20 & 29 & 33.24 & 0 & 599 & 1987.490 & 4.26 & 0.40 & 0.06 & 112.05 & 15.03 & 12.76 & 12.90 & 10.98 & 10.43 & 9.83 & 9.53 \\
\hline $1-4086$ & 21 & 22 & 58.085 & -47 & 22 & 17.44 & 0 & 236 & 1985.766 & 6.88 & 0.23 & 0.06 & 160.84 & 13.85 & 12.79 & 12.80 & 12.34 & 12.28 & 11.88 & 11.81 \\
\hline $2-4137$ & 21 & 23 & 0.030 & -41 & 52 & 8.42 & 0 & 342 & 1986.590 & 5.17 & 0.20 & 0.03 & 205.25 & 18.04 & 16.13 & 16.20 & 14.45 & 13.14 & 12.65 & 12.45 \\
\hline $2-3552$ & 21 & 23 & 1.218 & -29 & 32 & 47.73 & 0 & 465 & 1981.750 & 13.67 & 0.21 & 0.01 & 152.61 & 20.21 & 17.89 & 18.16 & 16.20 & 14.62 & 14.13 & 13.97 \\
\hline $2-570$ & 21 & 23 & 1.697 & -24 & 57 & 35.04 & 0 & 530 & 1989.760 & 4.90 & 0.37 & 0.02 & 84.21 & 19.24 & 17.04 & 17.17 & 0.00 & 13.55 & 13.05 & 12.79 \\
\hline $1-4087$ & 21 & 23 & 3.648 & -58 & 13 & 0.61 & 0 & 145 & 1984.653 & 6.13 & 0.30 & 0.04 & 128.46 & 15.07 & 13.01 & 13.04 & 11.44 & 10.40 & 9.76 & 9.49 \\
\hline $1-4088$ & 21 & 23 & 6.598 & -50 & 2 & 21.56 & 0 & 236 & 1985.766 & 6.88 & 0.18 & 0.01 & 111.55 & 18.27 & 15.69 & 15.98 & 13.82 & 12.65 & 12.04 & 11.79 \\
\hline $2-86$ & 21 & 23 & 7.295 & -28 & 9 & 48.46 & 0 & 465 & 1981.750 & 13.67 & 0.68 & 0.02 & 133.55 & 16.64 & 14.44 & 14.67 & 12.59 & 11.06 & 10.52 & 10.25 \\
\hline $2-1016$ & 21 & 23 & 7.781 & -20 & 10 & 55.38 & 0 & 599 & 1987.490 & 4.26 & 0.31 & 0.02 & 206.11 & 18.62 & 16.64 & 16.61 & 15.28 & 14.26 & 13.73 & 13.47 \\
\hline $2-2872$ & 21 & 23 & 17.080 & -43 & 52 & 42.13 & 0 & 287 & 1989.740 & 3.88 & 0.22 & 0.04 & 129.37 & 17.33 & 15.23 & 15.28 & 13.69 & 12.39 & 11.86 & 11.62 \\
\hline $2-4742$ & 21 & 23 & 18.438 & -24 & 49 & 13.74 & 0 & 530 & 1989.760 & 4.90 & 0.19 & 0.03 & 159.12 & 17.61 & 15.70 & 15.86 & 14.49 & 13.42 & 12.78 & 12.62 \\
\hline $2-3986$ & 21 & 23 & 22.641 & -45 & 27 & 34.10 & 0 & 287 & 1989.740 & 3.88 & 0.20 & 0.05 & 136.91 & 16.51 & 14.23 & 14.42 & 12.20 & 11.04 & 10.45 & 10.20 \\
\hline $2-2429$ & 21 & 23 & 23.726 & -21 & 49 & 49.87 & 0 & 599 & 1987.490 & 4.26 & 0.23 & 0.06 & 146.15 & 15.85 & 13.43 & 13.57 & 11.28 & 10.48 & 9.96 & 9.65 \\
\hline $2-4958$ & 21 & 23 & 25.257 & -17 & 38 & 47.43 & 0 & 599 & 1987.490 & 4.26 & 0.19 & 0.06 & 190.88 & 16.27 & 14.04 & 14.14 & 12.32 & 11.55 & 10.92 & 10.69 \\
\hline $1-4089$ & 21 & 23 & 31.292 & -70 & 25 & 38.36 & 0 & 74 & 1985.411 & 8.28 & 0.23 & 0.02 & 140.25 & 20.49 & 18.18 & 18.44 & 17.51 & 16.22 & 15.81 & 15.64 \\
\hline $1-4090$ & 21 & 23 & 31.339 & -70 & 25 & 38.00 & 1 & 75 & 1984.650 & 6.15 & 0.20 & 0.02 & 147.68 & 20.28 & 18.19 & 18.26 & 17.28 & 16.22 & 15.81 & 15.64 \\
\hline $1-4091$ & 21 & 23 & 33.809 & -68 & 11 & 11.29 & 0 & 75 & 1984.650 & 6.15 & 0.33 & 0.03 & 110.00 & 17.98 & 15.71 & 15.80 & 13.62 & 12.18 & 11.61 & 11.29 \\
\hline $2-588$ & 21 & 23 & 37.183 & -41 & 27 & 19.66 & 0 & 342 & 1986.590 & 5.17 & 0.37 & 0.03 & 195.15 & 19.09 & 16.98 & 17.06 & 16.17 & 15.23 & 14.79 & 14.47 \\
\hline $2-4814$ & 21 & 23 & 45.081 & -19 & 52 & 56.74 & 0 & 599 & 1987.490 & 4.26 & 0.19 & 0.02 & 93.70 & 19.66 & 17.21 & 17.27 & 14.79 & 13.33 & 12.73 & 12.47 \\
\hline $2-3636$ & 21 & 23 & 46.792 & -42 & 33 & 38.94 & 0 & 287 & 1989.740 & 3.88 & 0.20 & 0.07 & 142.33 & 16.20 & 14.15 & 14.19 & 12.86 & 12.12 & 11.56 & 11.32 \\
\hline $2-315$ & 21 & 23 & 48.695 & -44 & 46 & 3.88 & 0 & 287 & 1989.740 & 3.88 & 0.45 & 0.07 & 125.50 & 15.51 & 13.44 & 13.59 & 11.95 & 11.19 & 10.60 & 10.39 \\
\hline $1-4092$ & 21 & 23 & 50.463 & -64 & 54 & 30.75 & 0 & 107 & 1988.689 & 8.00 & 0.22 & 0.05 & 124.58 & 15.09 & 13.01 & 13.11 & 11.85 & 10.98 & 10.34 & 10.13 \\
\hline $2-1839$ & 21 & 23 & 50.544 & -19 & 11 & 45.83 & 0 & 599 & 1987.490 & 4.26 & 0.25 & 0.05 & 129.72 & 16.68 & 14.52 & 14.59 & 13.32 & 12.59 & 12.01 & 11.85 \\
\hline $1-4093$ & 21 & 23 & 51.770 & -65 & 34 & 31.11 & 0 & 107 & 1988.689 & 8.00 & 0.19 & 0.04 & 158.81 & 14.14 & 13.08 & 13.09 & 12.59 & 11.99 & 11.60 & 11.48 \\
\hline $1-4094$ & 21 & 23 & 52.780 & -67 & 26 & 56.49 & 0 & 75 & 1984.650 & 6.15 & 0.29 & 0.04 & 153.62 & 22.01 & 19.16 & 19.24 & 17.62 & 16.44 & 16.02 & 15.37 \\
\hline $1-4095$ & 21 & 23 & 54.068 & -73 & 21 & 50.79 & 0 & 47 & 1985.524 & 6.74 & 0.21 & 0.02 & 186.01 & 17.36 & 15.70 & 15.59 & 14.75 & 13.93 & 13.35 & 13.20 \\
\hline
\end{tabular}


Table 2. Relative average completeness at different Galactic latitudes assuming $95 \%$ completeness for $|b| \geq 35^{\circ}$.

\begin{tabular}{ccc}
\hline \hline$|b|$ & Average No. of stars per field & \% Completeness \\
\hline 20 & 21 & 42 \\
25 & 27 & 53 \\
30 & 40 & 80 \\
$\geq 35$ & 48 & 95 \\
\hline
\end{tabular}

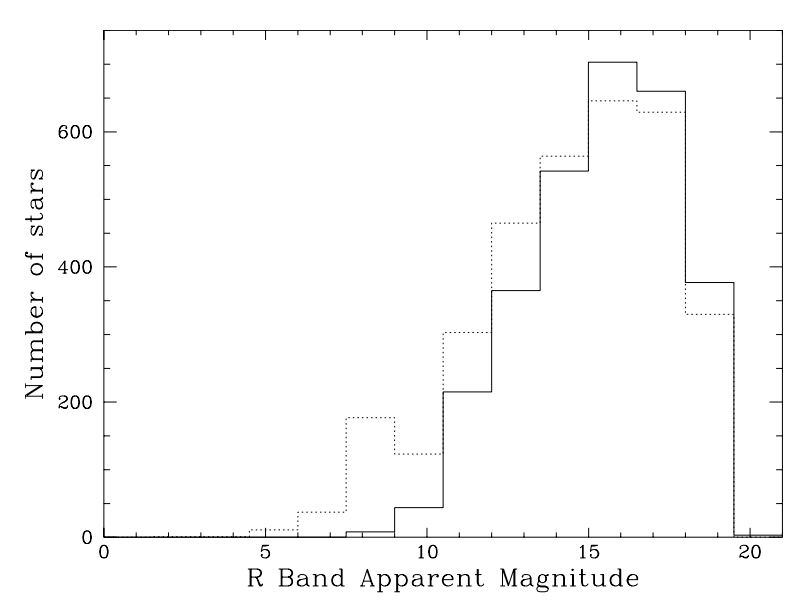

Fig. 8. Number of high proper motion stars per 1.5 magnitudes in $R$ for $-32.5^{\circ} \leq$ Dec $\leq-17.5^{\circ}$ and $|b| \geq 40^{\circ}$ : Solid line - this catalogue, dashed line - NLTT.

commonly quoted $S N R=10\left(\sigma_{m}<0.11\right)$ limits are $J=16.1$, $H=15.2$ and $K_{\mathrm{S}}=14.9$.

In order to allow a positional match with the 2MASS catalogue, we therefore applied proper-motion corrections to our catalogue coordinates to transform them to epoch 1999.5 (An approximate epoch for 2MASS (north or south) is $1999.5 \pm 2$ ). In order to search for 2MASS counterparts, we defined an error ellipse for each source that would cover its likely location on the 2MASS sky. The axes of these error ellipses were calculated as follows. We began with a base level representing the intrinsic positional uncertainty in the coordinates derived for both our survey and 2MASS. The main source of uncertainty in this base level comes from the possibility that some sources could be partly resolved multiple systems. Although 2MASS will initially flag a multiple system (e.g. a binary) with a $\sim 1$ arcsec separation as an extended source (using shape analysis; see 2MASS web-site http://www.ipac. caltech.edu/2mass), it may then subsequently remove it from the extended source catalogue (and put it in the point source catalogue) using additional shape analysis (e.g. symmetry metrics). Therefore, the 2MASS PSC will have some multiple sources with wider angular separation. The worst seeing accepted for 2MASS results in a FWHM of $\sim 4$ arcsec, and so it is possible that a binary with an angular separation of this order could appear in the PSC. The relative colours and brightnesses of the multiple components in such a system could result in optical and NIR source positions that differ by up to this level. This positional uncertainty dwarfs the astrometric uncertainties (up to 0.2 and 0.26 arcsec for our survey and 2MASS respectively), and we consequently set our base level uncertainty at this 4 arcsec value. In addition to this

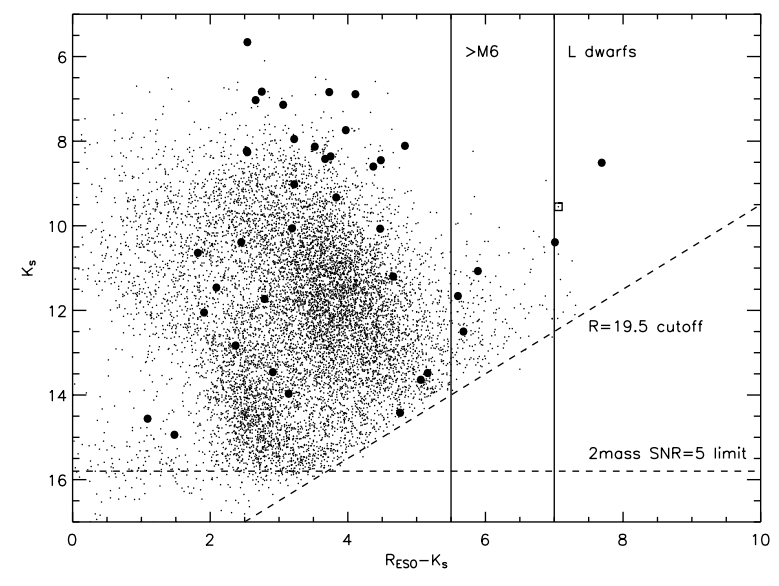

Fig. 9. Plot of $K_{\mathrm{S}}$ (2MASS) against $R_{\mathrm{ESO}}-K_{\mathrm{S}}$ for all stars with $K_{\mathrm{S}}$ magnitudes. Vertical solid lines indicate the $R_{\mathrm{ESO}}-K_{\mathrm{S}}$ limits for the stellar types indicated. The horizontal dashed line indicates the $S N R=5$ limit for the 2MASS $K_{\mathrm{S}}$ magnitude, the diagonal dashed line indicates the $R=19.5$ mag cutoff of our catalogue. Large points are stars with $\mu \geq$ $1.0^{\prime \prime} \mathrm{yr}^{-1}$. The square indicates the position of the benchmark brown dwarf LP 944-20 (Tinney 1998).

base level, there are the uncertainties associated with our proper motion measurements. We duly multiplied these proper motion uncertainties (in RA and Dec) by the difference between the epoch of their observation and $1999.5 \pm 2$ (to cover the full 2MASS base-line), and combined the results with the base level value to give final error ellipse axis in both coordinates for each source. We then searched for 2MASS sources within the error ellipses of our transformed catalogue coordinates using the GATOR tool provided by the Infrared Science Archive (IRSA). We added the stipulations that a $2 \mathrm{MASS}$ source must be at least 6 arcsec away from its nearest neighbour (to avoid spatial confusion from line-of-site proximity), and that it has not been flagged as a contamination/confusion affected source. Where multiple matches were found, the 2MASS counterpart nearest to the centre of the ellipse was selected.

In order to check for possible mis-matches amongst the highest proper motion sources, we carried out the following test. We selected the 38 highest proper motion sources ( $>1$ arcsec/yr) from our catalogue, and cross-matched them with 2 MASS as before, but this time with the added stipulation that a 2 MASS source must be redder than $J-K_{\mathrm{S}}=1.0$ to provide a match. This tested the possibility that some high proper motion red sources (most likely nearby late $\mathrm{M} / \mathrm{L}$ dwarfs) were being missed by chance alignment with bluer background sources. However, no additional 2MASS matches were found that had not already been identified without the colour stipulation. Our 2MASS matching procedure obtained 2MASS 


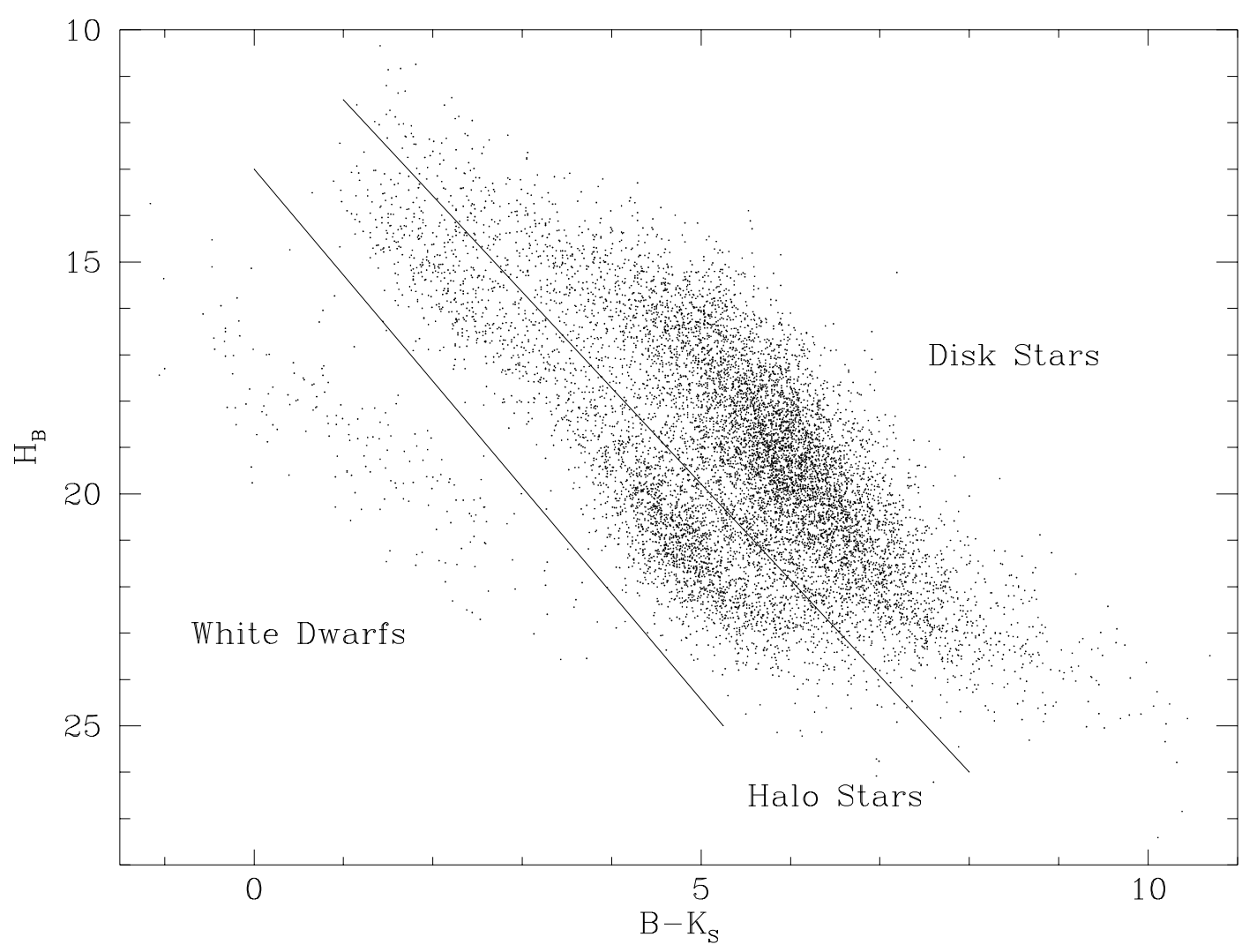

Fig. 10. Plot of reduced proper motion, $H_{B}$, against $B-K_{\mathrm{S}}$ for all stars in the survey with $B$ and $K_{\mathrm{S}}$ magnitudes. The lines showing the different regions of the plot are for guidance only.

photometry for 10870 of the 11899 sources in our catalogue, or $\sim 92 \%$.

Figure 9 is the $K_{\mathrm{S}}$ versus $R_{\mathrm{UK}}-K_{\mathrm{S}}$ colour-magnitude diagram for all stars in the catalogue with $2 \mathrm{MASS} K_{\mathrm{S}}$ magnitudes, and demonstrates the ability of this catalogue to identify late $\mathrm{M}$ and early L class brown dwarfs. Figure 10 is the $H_{B_{J}}$ versus $B_{J}-K_{\mathrm{S}}$ reduced proper motion diagram for the catalogue $\left(H_{B_{J}}=B+5+5 \log \mu\right.$ which can be shown to be the same as $H_{B_{J}}=B_{J}+5 \log V_{\mathrm{T}}-3.379$ where $V_{\mathrm{T}}$ is the tangential velocity of the star; PJH03).

\section{Discussion}

The main aims of high proper motion surveys are to preferentially select nearby stars and Halo stars and to differentiate between the Halo and Disk populations. As shown by Salim \& Gould (2002) and PJH03, optical-infrared reduced proper motion diagrams give much better differentiation of the disk, halo and white dwarf populations than optical-optical reduced proper motion diagrams. However, as passbands further into the infrared are used white dwarfs are lost due to their bluer colours (see Fig. 10), and the optimum reduced proper motion - passband combination for white dwarf selection seems to be $H_{B_{J}}$ versus $B-I$. Selection of objects near the Hydrogen burning limit (i.e. late $\mathrm{M}$ dwarfs and brown dwarfs) is best done with an optical-infrared colour magnitude diagram (see Fig. 9). Reliable differentiation of the various populations of stars is of great importance to studies of Galactic structure and dynamics, and the Halo luminosity function.
In PJH03 we used the $H_{B_{J}}, B-I$ and colour-colour plots to differentiate the disk and halo populations. Compared to Table 6 of Reid (1984) our numbers fell between those of models A and D and excluded models B and C. Although we have more than doubled the number of fields in the survey since that analysis the added fields were almost all at intermediate and low Galactic latitudes, and the predictions from Reid are for polar proper motion surveys. This means that we have added very few fields at high Galactic latitude, that the relative numbers of halo and disk stars at high latitude has not changed and that the new fields have therefore not made any difference to that result.

We have recently produced an extended version of the catalogue with a lower proper motion limit of $\mu \geq 0.1^{\prime \prime} \mathrm{yr}^{-1}$ and an $R$-band faint limit of $R \leq 21$ for the specific purpose of identifying proper motion binaries and potential moving group members. This extended version of the catalogue will not be made generally available because of the high relative uncertainties on the proper motions and the difficulty in characterizing the completeness that these limits produce. The fainter magnitude limit of the extended catalogue means that we will be able to use it to identify potential field brown dwarfs which would be too faint to appear in the basic catalogue. This is well demonstrated by the recovery of Kelu-1 in the extended catalogue with an $R$-band magnitude of $R_{\mathrm{UK}}=19.75$.

Acknowledgements. The authors would like to thank all of the staff of the SuperCOSMOS project at the ROE and Mike Read 
for producing Fig. 1. R.S.P. acknowledges support of a Liverpool John Moores University studentship. D.J.P. acknowledges support of a PPARC fellowship. The authors also would like to thank the referee, Dr. Ralf Scholz, for his extremely helpful comments on this paper.

\section{References}

Cabanela, J. E., Humphreys, R. M., Aldering, G., et al. 2003, PASP, 115,837

Carpenter, J. M. 2001, AJ, 121, 2851

Cruz, K. L., \& Reid, I. N. 2002, AJ, 123, 2828

Cutri, R. M., Skrutskie, M. F., van Dyk, S., et al. 2003, 2MASS All-Sky Catalog of Point Sources, University of Massachusetts and Infrared Processing and Analysis Center (IPAC/California Institute of Technology)

Gould, A., \& Salim, S. 2003, ApJ, 582, 1001

Hambly, N. C., Miller, L., MacGillivray, H. T., Herd, J. T., \& Cormack, W. A. 1998, MNRAS, 298, 897

Hambly, N. C., MacGillivray, H. T., Read, M. A., et al. 2001a, MNRAS, 326, 1279

Hambly, N. C., Irwin, M. J., \& MacGillivray, H. T. 2001b, MNRAS, 326, 1295

Hambly, N. C., Davenhall, A. C., Irwin, M. J., \& MacGillivray, H. T. 2001c, MNRAS, 326, 1315

Lepine, S., Rich, R. M., Neill, J. D., Caulet, A., \& Shara, M. M. 2002, ApJ, 581, 47
Lepine, S., Shara, M. M., \& Rich, R. M. 2003, AJ, 126, 921

Luyten, W. J. 1979a, LHS catalogue, University of Minnesota Luyten, W. J. 1979b, NLTT catalogue, University of Minnesota Persson, S. E., Murphy, D. C., Krzeminski, W., Roth, M., \& Rieke, M. J. 1998, AJ, 116, 2475

Pokorny, R. S., Jones, H. R. A., \& Hambly, N. C. 2003, A\&A, 397, 575

Reid, I. N., \& Cruz, K. L. 2002, AJ, 123, 2806

Reid, I. N., Cruz, K. L., Allen, P., et al. 2003, AJ, 126, 3007

Reid, I. N., Kilkenny, D., \& Cruz, K. L. 2002, AJ, 123, 2822

Reyle, C., Robin, A. C., Scholz, R.-D., \& Irwin, M. 2002, A\&A, 390, 491

Ribas, I. 2003, A\&A, 400, 297

Rojo, P. M., \& Ruiz, M. T. 2003, AJ, 126, 353

Ruiz, M. T., Wischnjewsky, M., Rojo, P. M., \& Gonzalez, L. E. 2001, ApJS, 133, 119

Salim, S., \& Gould, A. 2002, ApJ, 575, L83

Salim, S., \& Gould, A. 2003, ApJ, 582, 1011

Scholz, R.-D., Irwin, W., Ibata, R., Jahreiss, H., \& Malkov, O. Yu. 2000, A\&A, 353, 958

Scholz, R.-D., Lodieu, N., Ibata, R., et al. 2004, MNRAS, 347, 685

Silvestri, N. M., Oswalt, T. D., \& Hawley, S. L. 2002, AJ, 124, 1118

Tinney, C. G. 1998, MNRAS, 296, L42

Williams, D. B. 2000, JAVSO, 28, 12 